\title{
The Correlation Between Low Coagulation Factor XIII Activity and Anastomotic Leakage in Patients Who Undergo Bowel Resection
}

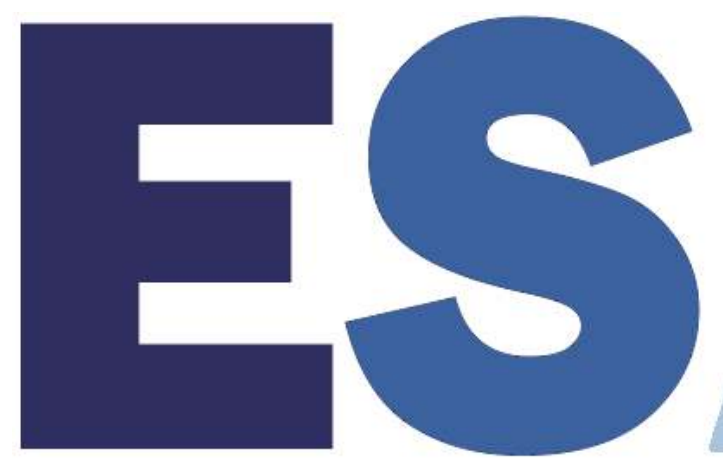

Mueller J.S., Chiari A., Herbst F., Dauser B., Korzeniewski T.

Hospital of St. John of God, Dept. of Anaesthesiology \& Intensive Care, Vienna

\section{Factor XIII - Anastomotic leakage}

- Factor XIII is crucial for blood clotting and wound healing

- Anastomotic leakages occur in around 5 percent of bowel resections postoperatively

- Several studies suggest a correlation between low coagulation factor XIII and anastomotic leakages after bowel resection

\section{Methodology - Prospective observational study}

- For six months, factor XIII activity in patients who underwent bowel resection was monitored preoperatively, on the first postoperative day and on the fifth postoperative day

- Patients were then divided into two groups (No leakage, Leakage) and the factor XIII activities measured were compared between the groups

\section{Results - Correlation}

Preoperative

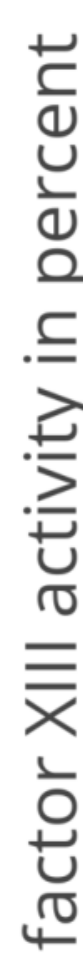

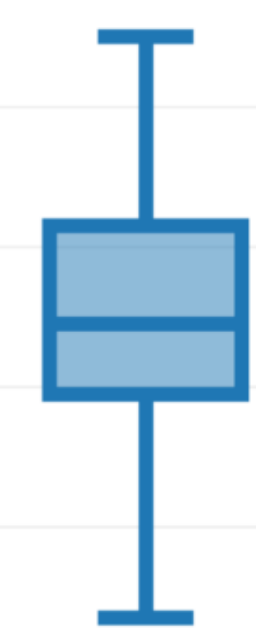

- No leakage $(n=74)$ - Leakage $(n=4)$

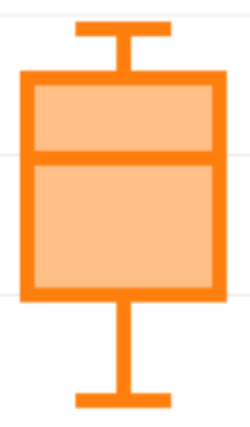

No leakage Leakage

$p=0.213$
First postoperative day

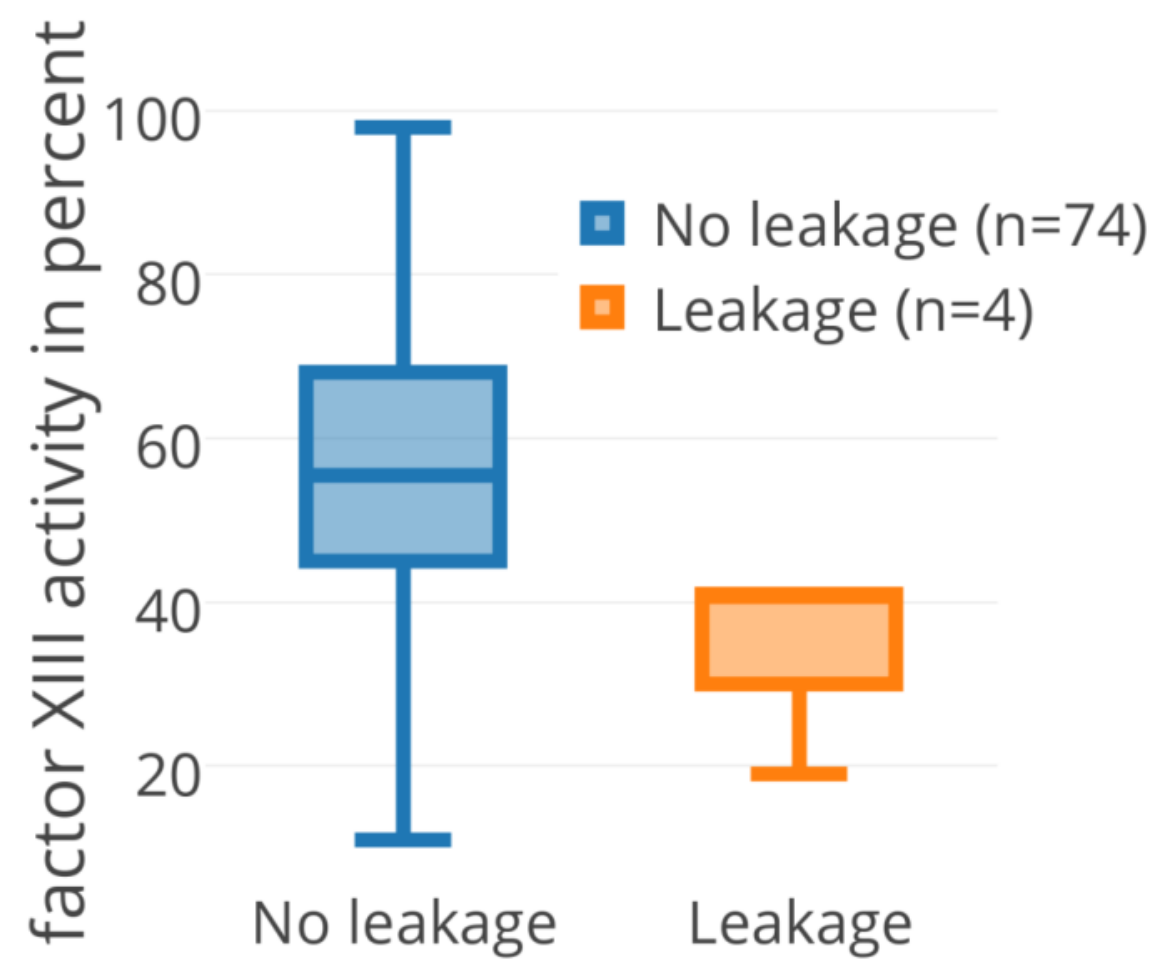

$p=0.013$
Fifth postoperative day

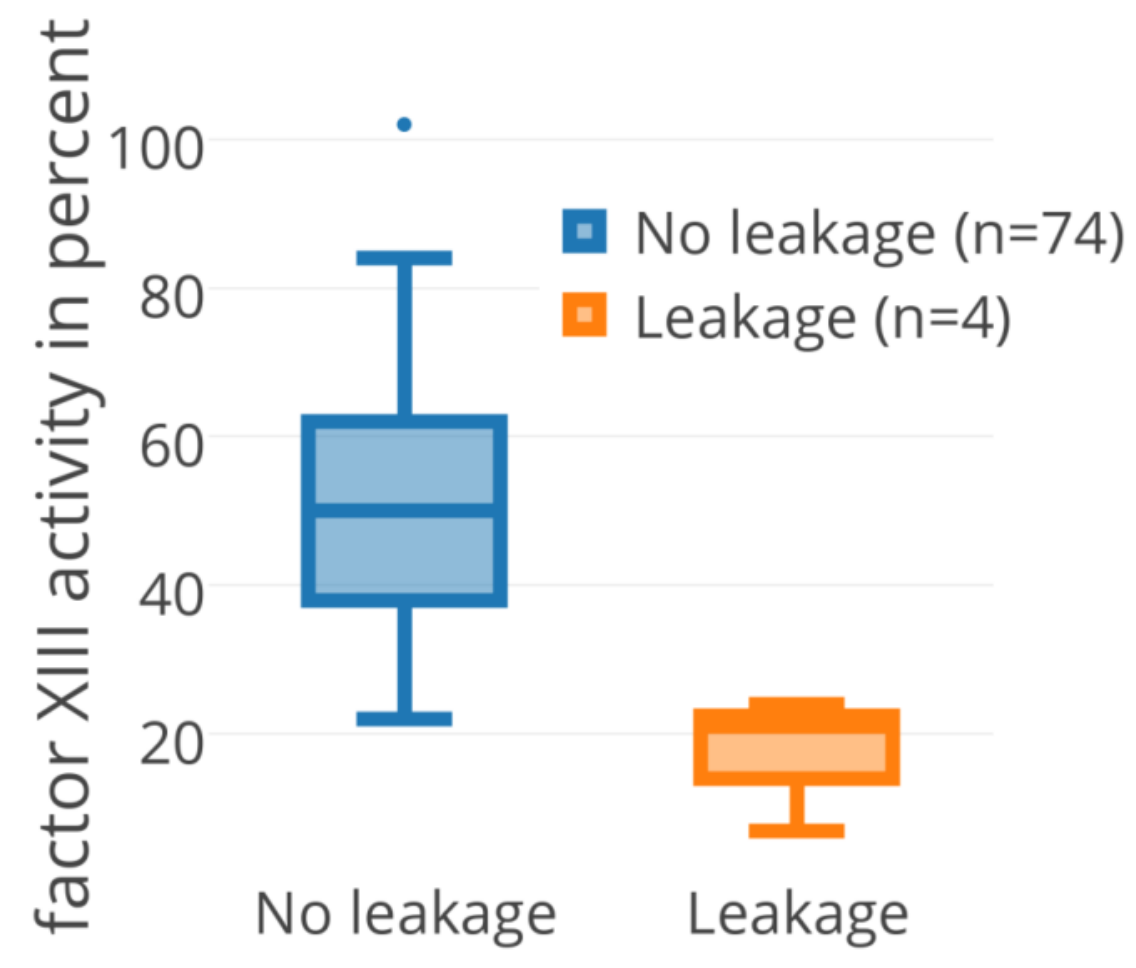

$p=0.01$

-In the "leakage"-group factor XIII activity was significantly lower than in the "noleakage"-group on the first and fifth postoperative day

- Factor XIII activity was also lower preoperatively but our study does not show a statistically significant correlation

\section{Discussion - Just a marker or the reason?}

- Anastomotic leakages normally occur around the fifth postoperative day. Since factor XIII activity is lower on the first postoperative day already there is a predictive component - Low factor XIII activity might be a reason for anastomotic leakages

-We plan to conduct further studies with larger samples and also want to test whether factor XIII substitution is beneficial for patients undergoing bowel resection 